\title{
Supporting Information: The Interplay of Al and Mg Speciation in Advanced Mg Battery Electrolyte Solutions
}

Kimberly A. See ${ }^{\dagger}$ Karena W. Chapman ${ }_{, *}^{*}$ Lingyang Zhu, ${ }^{\dagger}$ Kamila M. Wiaderek, ${ }^{\ddagger}$ Olaf J. Borkiewicz, Christopher J. Barile, ${ }^{\dagger}$ Peter J. Chupas, ${ }^{*}$ and Andrew A. Gewirth ${ }^{* \dagger}$

${ }^{\dagger}$ Department of Chemistry, University of Illinois at Urbana-Champaign, Urbana, Illinois 61801 United States

${ }^{\ddagger}$ Xray Science Division, Advanced Photon Source, Argonne National Laboratory, Argonne, Illinois, 60439, United States

Table S1. Gaussian fit parameters of the XPDF data.

\begin{tabular}{|c|c|c|c|}
\hline & Peak center $(\AA)$ & Area $(\mathrm{G}(\mathrm{r}) \cdot \mathrm{r})$ & Area (normalized) \\
\hline \multirow[t]{6}{*}{$0.06 \mathrm{M} \mathrm{MgCl}_{2}$} & 2.38 & 0.036 & 1.0 \\
\hline & 3.27 & 0.069 & 1.9 \\
\hline & 4.07 & 0.0076 & 0.21 \\
\hline & 4.43 & 0.038 & 1.0 \\
\hline & 4.82 & 0.038 & 1.1 \\
\hline & 5.32 & 0.045 & 1.3 \\
\hline \multirow[t]{7}{*}{$0.03 \mathrm{M} \mathrm{AlCl}_{3}$} & 2.19 & 0.062 & 1.0 \\
\hline & 2.95 & 0.025 & 0.41 \\
\hline & 3.43 & 0.020 & 0.32 \\
\hline & 3.78 & 0.049 & 0.78 \\
\hline & 4.51 & 0.042 & 0.68 \\
\hline & 4.88 & 0.035 & 0.56 \\
\hline & 5.34 & 0.038 & 0.61 \\
\hline \multirow[t]{7}{*}{ MACC, as-prep. } & 2.13 & 0.049 & $1.0 / 2.0$ \\
\hline & 2.37 & 0.025 & $0.50 / 1.0$ \\
\hline & 3.25 & 0.054 & $1.1 / 2.2$ \\
\hline & 3.47 & 0.059 & $1.2 / 2.4$ \\
\hline & 4.46 & 0.070 & $1.4 / 2.8$ \\
\hline & 4.88 & 0.056 & $1.1 / 2.3$ \\
\hline & 5.42 & 0.073 & $1.5 / 2.9$ \\
\hline \multirow[t]{7}{*}{ MACC, cond. } & 2.15 & 0.024 & $1.0 / 0.47$ \\
\hline & 2.39 & 0.050 & $2.1 / 1.0$ \\
\hline & 3.27 & 0.079 & $3.3 / 1.6$ \\
\hline & 3.49 & 0.038 & $1.6 / 0.76$ \\
\hline & 4.39 & 0.079 & $3.3 / 1.6$ \\
\hline & 4.86 & 0.068 & $2.9 / 1.4$ \\
\hline & 5.35 & 0.090 & $3.8 / 1.8$ \\
\hline
\end{tabular}




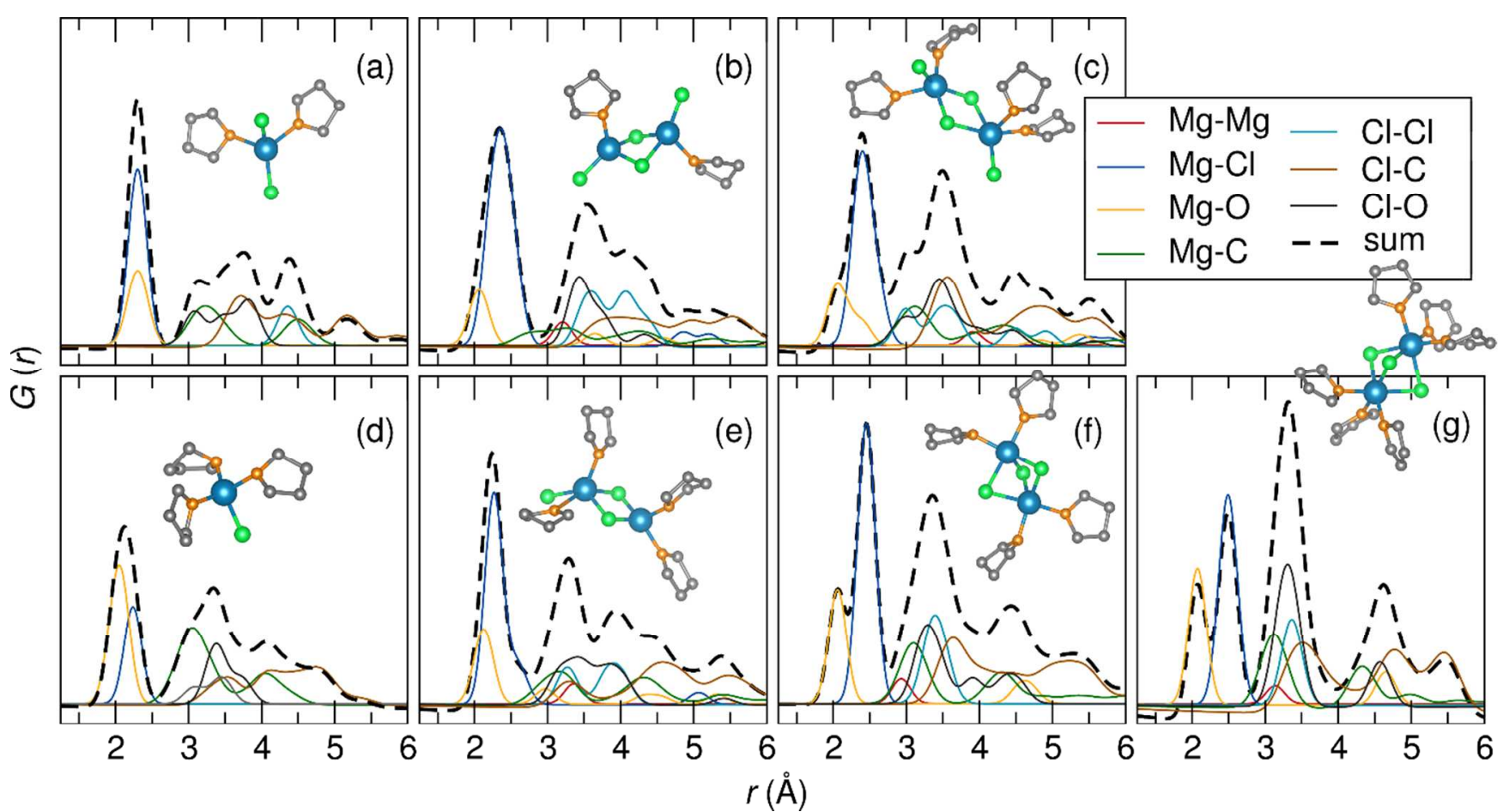

Figure S1. Simulated Xray PDF patterns of possible Mg complexes in THF. The PDF is simulated with PDFgui using only the complex with the solvent THF removed. (a-c) represent neutral complexes while $(\mathrm{d}-\mathrm{g})$ have a nominal charge of +1 . The complexes were originally calculated in the (a) liquid, (b) liquid, (c) gas, (d) liquid, (e) liquid, (f) gas, and (g) solid phase.

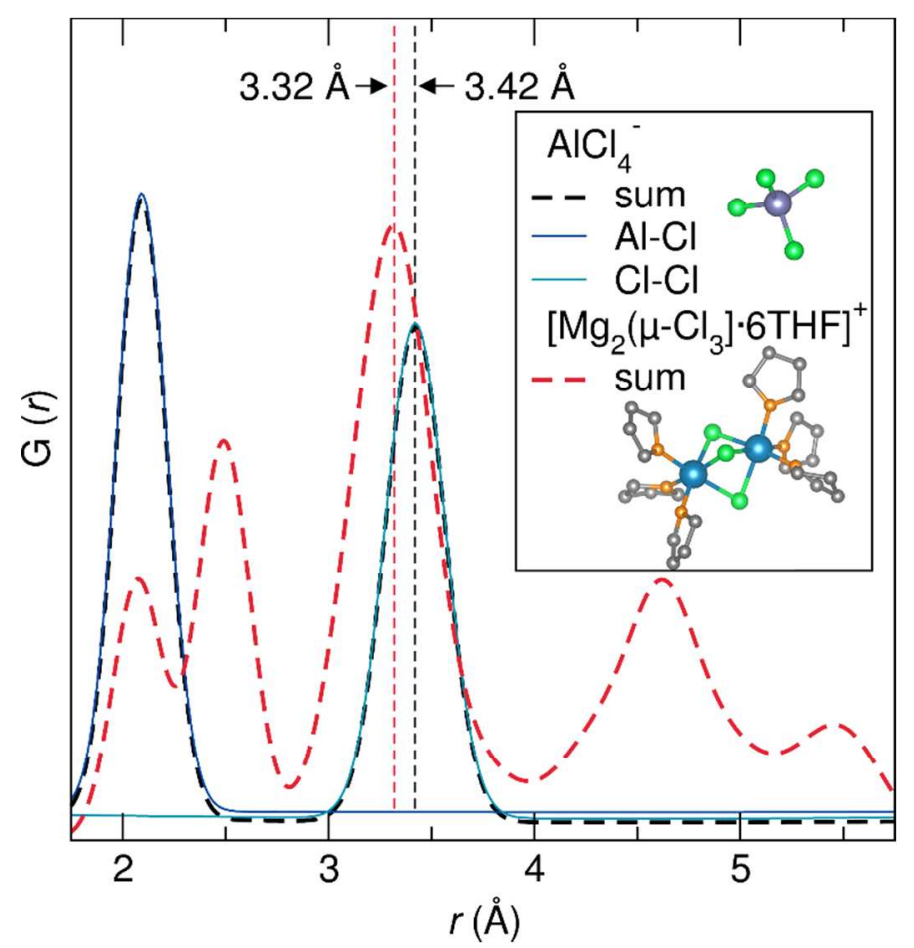

Figure S2. Simulated Xray PDF patterns of $\mathrm{AlCl}_{4}^{-}$and the octahedrally coordinated $\mathrm{Mg}$ dimer complex $\left[\mathrm{Mg}_{2}\left(\mu-\mathrm{Cl}_{3}\right) \cdot \mathrm{THF}_{6}\right]^{+}$. 


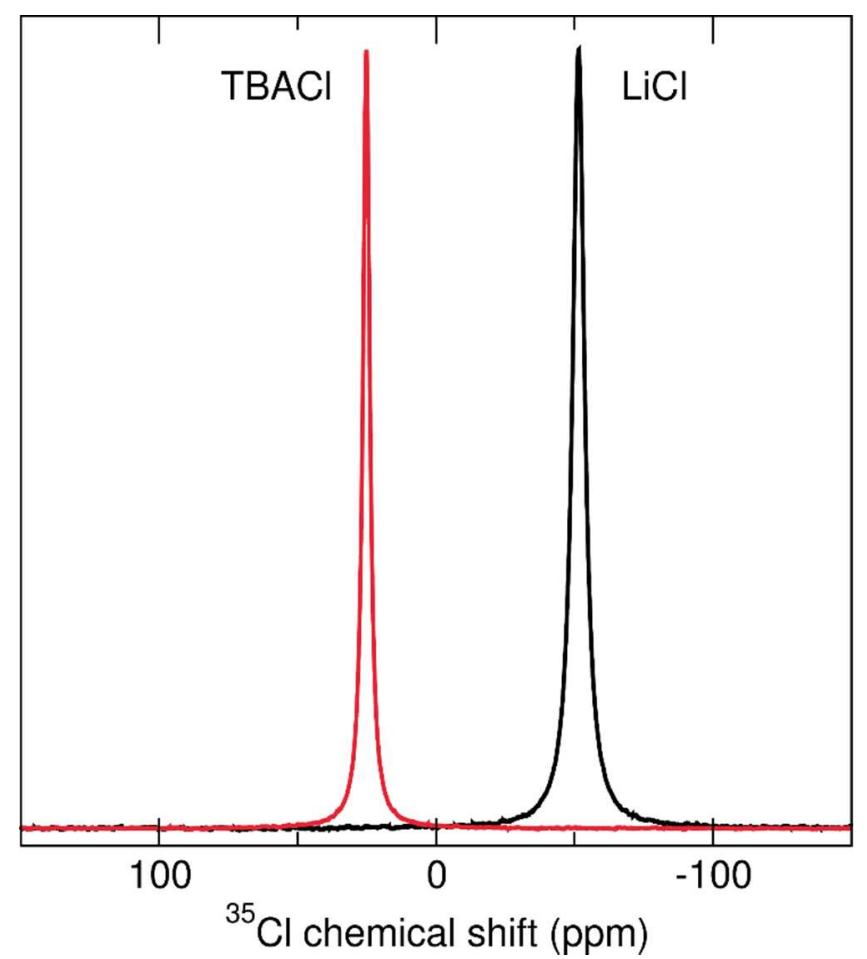

Figure S3. ${ }^{35} \mathrm{C}$ NMR of $0.21 \mathrm{M}$ solutions of tetrabutylammonium chloride (TBACl) and lithium chloride (LiCl) in THF. The intensity is normalized. 